\title{
Inhibitory Effects of Beraprost Sodium in Murine Hepatic Sinusoidal Obstruction Syndrome
}

\author{
MAKOTO NAKURA ${ }^{1}$, TOMOHARU MIYASHITA ${ }^{2}$, YASUHIKO YAMAMOTO ${ }^{3}$, SATOSHI TAKADA ${ }^{1}$, \\ SHUNSUKE KANOU $^{1}$, HIDEHIRO TAJIMA $^{1}$, HIROYUKI TAKAMURA ${ }^{2}$ and TETSUO OHTA ${ }^{1}$ \\ ${ }^{1}$ Department of Gastroenterological Surgery, Kanazawa University Hospital, Kanazawa, Japan; \\ ${ }^{2}$ Department of Surgical Oncology, Kanazawa Medical University Hospital, Kahoku, Japan; \\ ${ }^{3}$ Department of Biochemistry and Molecular Vascular Biology, Kanazawa \\ University Graduate School of Medical Sciences, Kanazawa, Japan
}

\begin{abstract}
Background/Aim: In this study, the liver sinusoidal endothelial cells (LSECS)-protective effects of beraprost sodium (BPS) were investigated using mice with monocrotaline (MCT)induced sinusoidal obstruction syndrome (SOS). Materials and Methods: The mice were divided into BPS, placebo and control groups. They were killed $48 \mathrm{~h}$ after MCT administration, and blood samples and liver tissues were evaluated. Immunostaining was performed using anti-SE-1 and anti$C D 42 b$ antibodies, whereas plasminogen activator inhibitor (PAI-1) and endothelial nitric oxide synthase (eNOS) levels were evaluated using western blot or real-time RT-PCR. Results: On pathological examination, SOS-related findings were observed in zone 3 in the placebo group; however, these were significantly suppressed in the BPS group. SE-1 staining showed a consistent number of LSECs in the BPS group compared with that in the placebo group, while CD42b staining showed a significant decrease in the number of extravasated platelet aggregation (EPA) in the BPS group. PAI1 expression was significantly lower in the BPS group than in the placebo group; however, eNOS expression was significantly higher in the BPS group than in the placebo group. Conclusion: Prophylactic administration of BPS is useful for suppressing the development of SOS through the protective effects of LSEC.
\end{abstract}

This article is freely accessible online.

Correspondence to: Tomoharu Miyashita, MD, Ph.D., Department of Surgical Oncology, Kanazawa Medical University Hospital, 1-1 Daigaku, Uchinada, Kahoku, Ishikawa 920-0293, Japan. Tel: +81 762862211, Fax: +81 762864626, e-mail: tomoharumiya@gmail.com

Key Words: Beraprost sodium, liver sinusoidal endothelial cells, liver transplantation, oxaliplatin based chemotherapy, sinusoidal obstruction syndrome, veno-occlusive disease.
Sinusoidal obstruction syndrome (SOS), formally known as veno-occlusive disease (VOD) (1), is defined as a syndrome that involves symptoms such as abdominal pain, hepatomegaly, ascites, weight gain, portal hypertension, and jaundice (2). Once the onset of SOS/VOD has occurred, it is life-threatening, with a mortality rate of at least $80 \%(3,4)$. Reported causes of SOS/VOD include colorectal cancer chemotherapy containing L-OHP, acute leukemia chemotherapy, hematopoietic stem cell transplantation and liver transplantation (5-8).

In a clinical study, it was reported that defibrotide was effective for treating SOS/VOD; however, its use has not been approved in Japan (9). Other studies suggest that antithrombin III, prostaglandin E1, low-molecular-weight heparin, and ursodeoxycholic acid are useful alternatives (10-13). Although various treatment options have been reported, no specific treatment has been established as effective for treating SOS/VOD to date (2).

We had previously classified three phases, from liver sinusoidal endothelial cell (LSEC) damage to organ dysfunction, utilizing the predicted pathogenic mechanism of SOS (14). The first phase was detachment of the LSECs, and sinusoidal wall destruction after LSEC injury by immunosuppressant and chemotherapeutic agents. The second phase was extravasated platelet aggregation (EPA) accomplished by sinusoidal wall destruction. The various growth factors, including thromboxane A2 (TXA2), serotonin, transforming growth factor-beta (TGF- $\beta$ ) and plasminogen activator inhibitor-1 (PAI-1), were released via EPA in the space of Disse in zone 3; portal hypertension and the progression of hepatic fibrosis were induced. The third phase was organ dysfunction following portal hypertension, hepatic fibrosis, and suppressed liver regeneration through the various growth factors secreted via EPA (14-17). It is therefore considered that protection of the LSECs is an important measure against SOS/VOD $(14,18)$. 
Beraprost sodium (BPS) is a prostaglandin $\mathrm{I}_{2}\left(\mathrm{PGI}_{2}\right)$ derivative used to treat both chronic arterial occlusive disease and primary pulmonary hypertension. In addition to its antiplatelet and vasodilatory effects, it has been reported to have protective effects on vascular endothelial cells (19, 20). The aim of this study was thus to investigate the prophylactic effects of BPS on SOS/VOD and its LSECprotective effects using a murine model with monocrotaline (MCT)-induced SOS.

\section{Materials and Methods}

Animals. Five-week-old female $\mathrm{Crl}$ :CD1(ICR) mice weighing between 20 and $30 \mathrm{~g}$ were purchased from Charles River Laboratories Japan, Inc. (Yokohama, Japan) (18). All experiments included in this study were submitted to the Kanazawa University Animal Experiments Committee in accordance with the Animal Experiments Protocol, and approval was obtained (approval no.: AP-153599).

Experimental protocol. MCT was purchased in powder form from FUJIFILM Wako Pure Chemical Corporation (Tokyo, Japan). Free samples of BPS in powder form were provided by Toray Industries, Inc. (Chiba, Japan) for research purposes.

After two weeks of rearing with standard food, the mice were recruited into the study at the age of seven weeks. They had ad libitum access to drinking water and were fasted for twelve hours before drug administration.

There were three group allocations ( $\mathrm{n}=10$ in each group); with reference to a previous report (18), $270 \mathrm{mg} / \mathrm{kg}$ MCT was administered intraperitoneally to prepare the SOS model. The BPS group was administered $200 \mu \mathrm{g} / \mathrm{kg}$ BPS intraperitoneally one hour before and both three and nine hours after MCT administration. Following the same regimen as the BPS group, the placebo group was administered physiological saline solution instead of BPS; the control group was administered physiological saline solution in place of both MCT and BPS. The mice were killed $48 \mathrm{~h}$ after MCT administration, and blood and liver samples were collected. The liver was visually inspected, and the presence or absence of ascites were determined.

Pathological assessment. Liver tissue was fixed with $10 \%$ neutral buffered formalin, embedded in paraffin, and sectioned at a thickness of $4 \mu \mathrm{m}$. Hematoxylin and eosin stains were prepared and examined microscopically. In connection with SOS scoring, sinusoidal dilation, sinusoidal congestion, endothelial damage of the central vein, and necrosis were evaluated $(17,21)$. For each of these parameters, a score between 0 and 3 was given ( 0 : normal; 1 : mild; 2: moderate; and 3: severe). The sum of the parameter scores was determined as SOS scores, and evaluations were performed on the basis of the mean for each group.

Immunohistochemical staining. LSEC damage was evaluated by immunostaining with the following antibodies: (i) SE-1, against hepatic sinusoidal endothelial cells (NB110-68095; Novus Biologicals, Cambridge, UK); (ii), against glycoprotein Ib- $\alpha$ (orb6179, cluster of differentiation 42b; Biorbyt, Ltd., Cambridge, UK); and (iii) against caspase-3 (\#9664, Cell Signaling Technology, Inc., Tokyo, Japan).
The space of Disse originally contains reticular fibers, which consist primarily of Type III collagen. When EPA occurs, Type III collagen and platelet aggregates are formed in the space of Disse; one effect of this is increased Type I collagen production by hepatic stellate cells. These activities lead to further occlusion in the space of Disse. Collagen fibers in zone 3 were evaluated using Azan staining (Muto pure chemicals, Tokyo, Japan), which stains collagen fibers deep blue, and silver-plating stain, which stains reticular fibers composed of Type III collagen.

Each staining was measured for the area stained (\%) as the average of five randomly selected images in the center of zone 3 using Image $\mathbf{J}$ (National Institutes of Health, Bethesda, MD, USA).

Western blot analysis. Plasminogen activator inhibitor-1 (PAI-1) is a marker for vascular endothelial cell damage, while endothelial nitric oxide synthase (eNOS) is a marker for LSEC-protective effects. These were evaluated via western blot analysis using antiPAI-1 (D9C4) rabbit monoclonal antibody (\#11907; Cell Signaling Technology, Inc., Tokyo, Japan), and anti-eNOS antibody (\#9572; Cell Signaling Technology, Inc., Tokyo, Japan), diluted 1,000x.

Real-time reverse-transcription polymerase chain reaction (realtime RT-PCR). Liver samples were collected from the mice, and total RNA was purified using the RNeasy Plus Mini Kit (Qiagen Co., Ltd., Tokyo, Japan). Real-time RT-PCR was carried out using Multiplex Quantitative PCR Systems (Mx3000P; Agilent Technologies, Inc. Santa Clara, CA, USA). In addition to PAI-1 and eNOS, expressions of Akt-1 in the PI3K/Akt pathway and phosphatidylinositol-3 kinase (PI3K) at an upstream position were numerically expressed and evaluated.

Hematological findings. White blood cells (WBCs), platelet count, aspartate aminotransferase (AST), alanine aminotransferase (ALT), lactate dehydrogenase (LDH), total bilirubin (T-Bil), and hyaluronic acid were evaluated.

Statistical analysis. Results were expressed as mean \pm standard deviation (SD). The intergroup differences between measured variables were evaluated using one-way analysis of variance followed by Bonferroni multiple-comparison method with the IBM SPSS 24.0 software (IBM Corp, Armonk, NY, USA). A p-value less than 0.05 was considered statistically significant.

\section{Results}

\section{Pathological signs}

Visual findings. The volume of ascites was high in the placebo group, and the liver had a deep red coloration. The findings in the BPS group were the same as those in the control group, with neither ascites nor discoloration (Figure 1A). Physiological saline solution infusion via the portal vein resulted in resistance in the placebo group, with a mild color change. In the BPS and control groups, there was little resistance, and the liver changed to white (Figure 1B).

\section{Histopathological findings}

Hematoxylin and eosin staining. In the placebo group, hematoxylin and eosin staining of the liver tissue revealed sinusoidal dilation and congestion around zone 3 in the 
central vein; these signs were mild in the BPS and control groups (Figure 1C).

SOS score. In the BPS group, the SOS score was significantly lower than in the placebo group (placebo: 9.10 \pm 1.37 ; BPS: $2.00 \pm 2.11 ; \mathrm{n}=10$ in each group; $p<0.0001$ ) (Table I).

\section{Immunohistochemical staining}

Evaluation of sinusoidal endothelial cells. Immunohistochemical analysis of the LSECs using an anti-SE-1 antibody confirmed that marked staining was found along the sinusoids in zone 3 surrounding the central vein in both the control and BPS groups; less staining was observed in the placebo group (control: 5.81 \pm 0.80 ; placebo: $0.80 \pm 0.31$; BPS: $10.98 \pm 1.05$; control vs. placebo: $p=0.00014$; placebo vs. BPS: $p<0.0001$; Figure 1D and 1G).

The presence of platelets was examined using CD42b staining. In the placebo group, extravasated platelets were present in the space of Disse in zone 3. Few extravasated platelets were found in the BPS group, and none were found in the control group (control: $0.074 \pm 0.035$; placebo: 4.33 \pm 0.73 ; BPS: $0.20 \pm 0.044$; control vs. placebo: $p=0.00062$; placebo vs. BPS: $p=0.00069$; Figure $1 \mathrm{E}$ and $1 \mathrm{H}$ ).

The apoptotic cells were examined using caspase- 3 staining. Caspase- 3 was expressed in the placebo group, centered in zone 3. Expression of caspase-3 was lower in the BPS group, and no expression was observed in the control group (control: $0.0040 \pm 0.0034$; placebo: $1.64 \pm 0.60$; BPS: $0.26 \pm 0.061$; control $v s$. placebo: $p=0.011$; placebo $v s$. BPS: $p=0.021$; Figure $1 \mathrm{~F}$ and $1 \mathrm{I})$.

Evaluation of connective tissue fibers. Azan staining of the hepatic fibrosis revealed that the staining centered around zone 3 in the placebo group was more intense than in the BPS group (control: 3.51 \pm 1.09 ; placebo: 12.89 \pm 3.35 ; BPS: $2.70 \pm 1.21$; control $v s$. placebo: $p=0.0057$; placebo $v s$. BPS: $p=0.0041$; Figure 2A and 2C).

On the other hand, the silver-plating stain centered around zone 3 in the placebo group was less intense than in the BPS group (control: 4.58 \pm 0.12 ; placebo: 1.21 \pm 0.29 ; BPS: $3.82 \pm 0.75$; control vs. placebo: $p<0.0001$; placebo $v s$. BPS: $p=0.0024$; Figure 2B and 2D).

\section{Evaluation of vascular endothelial cell damage}

Western blotting method. Expression of PAI-1 in the placebo group was higher than in both the BPS and control groups (Figure 3A).

Expression of eNOS in the BPS group was higher than in both the placebo and control groups (Figure 3B).

Real-time RT-PCR. The levels of expression of PAI-1, eNOS, Akt-1 and PI3K were represented numerically and compared; the value in the control group was set at 1.00 .
Table I. SOS score for H\&E staining at $48 \mathrm{~h}$ after euthanasia.

\begin{tabular}{lccc}
\hline Histopathological parameter & Placebo & BPS & $p$-Value \\
\hline Sinusoidal dilation & $2.0 \pm 0.8$ & $0.6 \pm 0.7$ & $<0.001$ \\
Sinusoidal congestion & $2.8 \pm 0.4$ & $0.6 \pm 0.8$ & $<0.001$ \\
Endothelial damage in the central vein & $2.4 \pm 0.7$ & $0.6 \pm 0.7$ & $<0.001$ \\
Hepatocellular necrosis & $1.9 \pm 0.7$ & $0.2 \pm 0.4$ & $<0.001$ \\
Total (SOS score) & $9.1 \pm 1.4$ & $2.0 \pm 2.1$ & $<0.001$ \\
\hline
\end{tabular}

Expression of PAI-1 in the placebo group was significantly higher than in the BPS and control groups (control: 1.00; placebo: 185.39; BPS: 0.23; control vs. placebo: $p=0.00088$; placebo vs. BPS: $p=0.00088$; Figure 3C).

Expression of eNOS in the BPS group was higher than in the placebo and control groups (control: 1.00; placebo: 0.16; BPS: 1.78 ; control vs. placebo: $p<0.0001$; placebo $v s$. BPS: $p=0.021$; Figure 3D).

Expression of Akt-1 in the placebo group was significantly lower than in the BPS and control groups (control: 1.00; placebo: 0.27; BPS: 1.09; control vs. placebo: $p<0.0001$; placebo vs. BPS: $p=0.004$; Figure 3E).

Expression of PI3K in the placebo group was significantly lower than in the BPS and control groups (control: 1.00; placebo: 0.25; BPS: 1.57; control vs. placebo: $p<0.0001$; placebo $v s$. BPS: $p=0.0018$; Figure $3 \mathrm{~F}$ ).

Blood chemistry test results. No significant intergroup differences were found between WBC counts (control: $23.7 \pm 8.6 \times 10^{2}$ cells $/ \mu$; placebo: $17.8 \pm 7.5 \times 10^{2}$ cells $/ \mu \mathrm{l}$; BPS: $20.8 \pm 8.1 \times 10^{2}$ cells $/ \mu \mathrm{l} ; \mathrm{n}=10$; control $v s$. placebo: $p=0.36$; placebo $v s$. BPS: $p=1.20)$.

The platelet count in the placebo group was significantly lower than in the BPS and control groups (control: $70.1 \pm 18.6 \times 10^{4} / \mu \mathrm{l}$; placebo: $4.84 \pm 3.21 \times 10^{4} / \mu \mathrm{l}$; $\mathrm{BPS}$ : $48.2 \pm 11.9 \times 10^{4} / \mu \mathrm{l} ; \mathrm{n}=10$; control vs. placebo: $p<0.0001$; placebo $v s$. BPS: $p<0.0001)$.

AST, ALT and LDH levels were significantly higher in the placebo group than in the BPS and control groups, as follows: (i) $[\mathrm{AST}]$ control: $75.2 \pm 17.3 \mathrm{IU} / \mu \mathrm{l}$; placebo: 1,013.3 $\pm 749.1 \mathrm{IU} / \mu \mathrm{l}$; BPS: $129.9 \pm 63.3 \mathrm{IU} / \mu \mathrm{l} ; \mathrm{n}=10$; control vs. placebo: $p=0.0010$; placebo $v s$. BPS: $p=0.014$; (ii) [ALT] control: $28.6 \pm 8.2 \mathrm{IU} / \mu \mathrm{l}$; placebo: $923.9 \pm 659.4 \mathrm{IU} / \mu \mathrm{l}$; BPS: $90.2 \pm 119.0 \mathrm{IU} / \mu \mathrm{l} ; \mathrm{n}=10$; control $v s$. placebo: $p=0.0060$; placebo vs. BPS: $p=0.0084$; and (iii) [LDH] control: 290.3 $\pm 115.3 \mathrm{IU} / \mu \mathrm{l}$; placebo: 2,477.6 $\pm 2,177.2 \mathrm{IU} / \mu \mathrm{l}$; BPS: $372.2 \pm 191.5 \mathrm{IU} / \mu \mathrm{l} ; \mathrm{n}=10$; control $v s$. placebo: $p=0.033$; placebo $v s$. BPS: $p=0.041$.

The hyaluronic acid level in the placebo group was significantly higher than in the BPS and control groups (control: 182.3 $\pm 50.2 \mathrm{IU} / \mu \mathrm{l}$; placebo: $2243.0 \pm 1135.9 \mathrm{IU} / \mu \mathrm{l}$; 

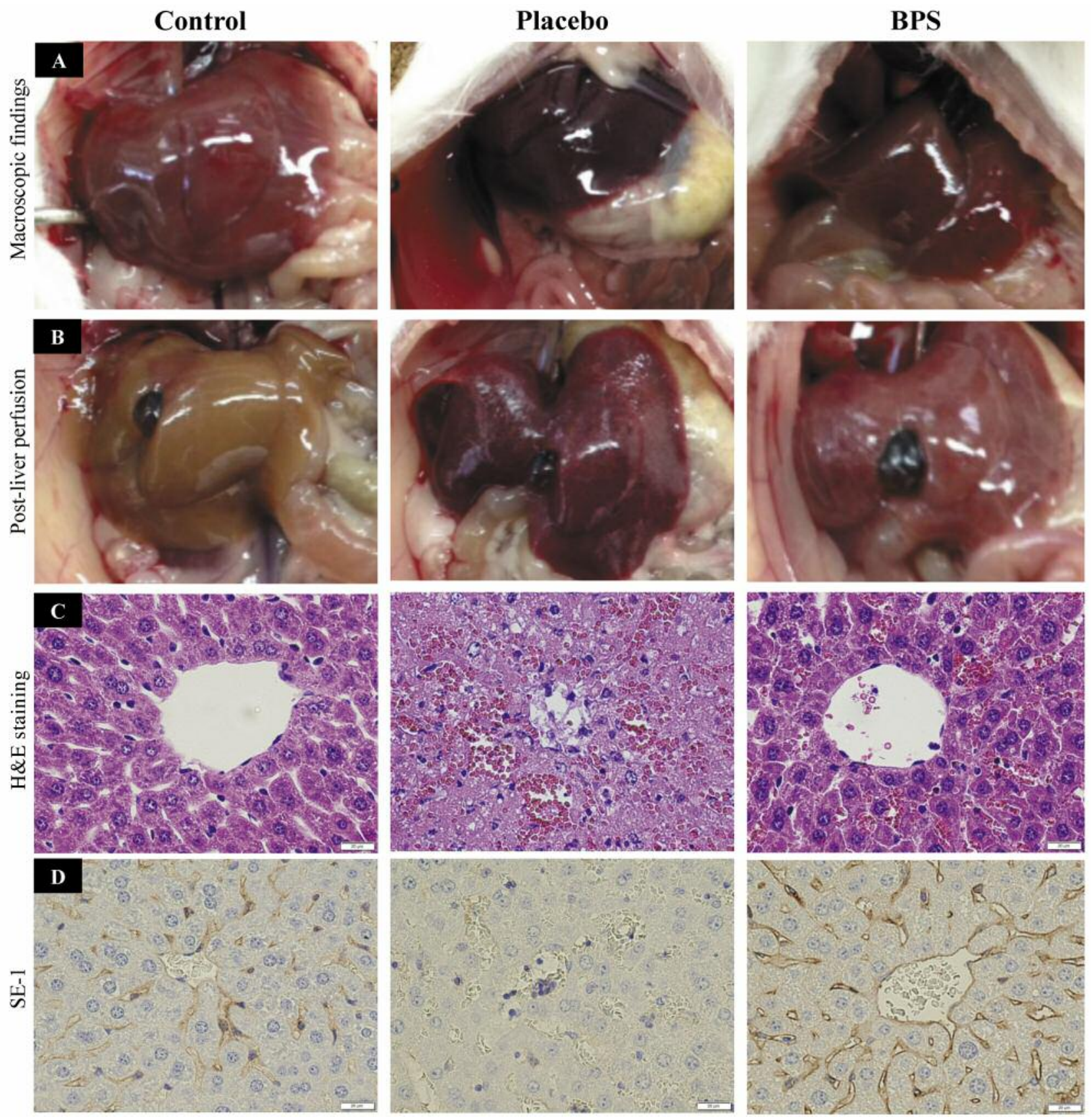

Figure 1. Continued

BPS: $670.6 \pm 184.3 \mathrm{IU} / \mu \mathrm{l} ; \mathrm{n}=10$; control $v s$. placebo: $p=0.00085$; placebo $v s$. BPS: $p=0.0058)$.

The T-Bil level in the placebo group was significantly higher than in the control group, but showed no difference in the BPS group (control: $0.066 \pm 0.011 \mathrm{IU} / \mu \mathrm{l}$; placebo: $0.155 \pm 0.026 \mathrm{IU} / \mu \mathrm{l}$; BPS: $0.131 \pm 0.030 \mathrm{IU} / \mu \mathrm{l} ; \mathrm{n}=10$; control vs. placebo: $p<0.0001$; placebo $v s$. BPS: $p=0.21$ ).

\section{Discussion}

This study clearly indicated that BPS administration can prevent SOS. In addition to inhibition of platelet aggregation, the suggested mechanisms of action of BPS include activation of the Akt-eNOS pathway mediated by $\mathrm{PI} 3 \mathrm{~K}$; increase in nitric oxide production, resulting in 

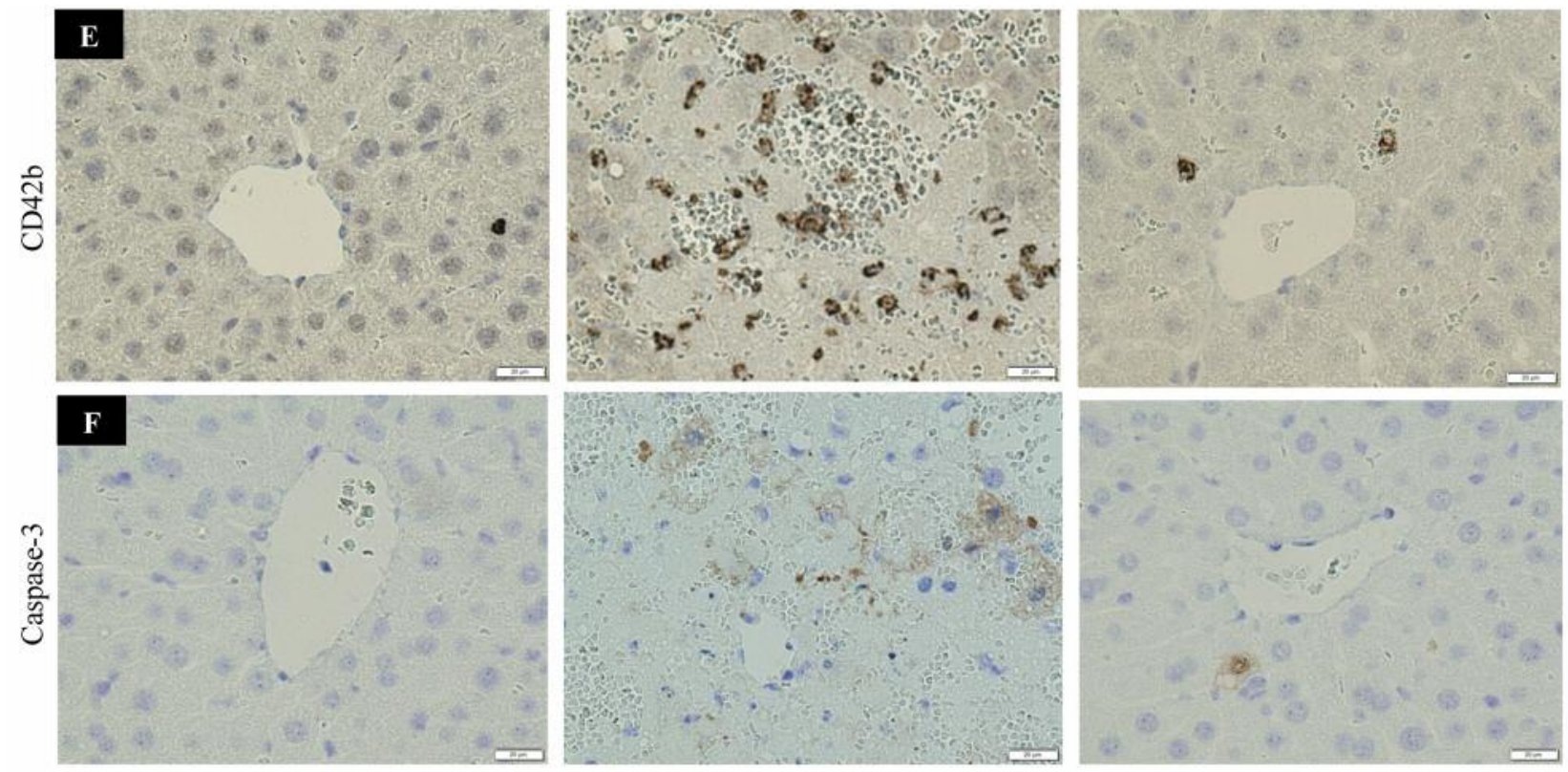

\section{G}

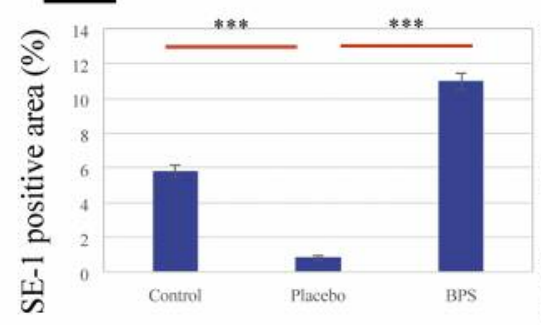

H

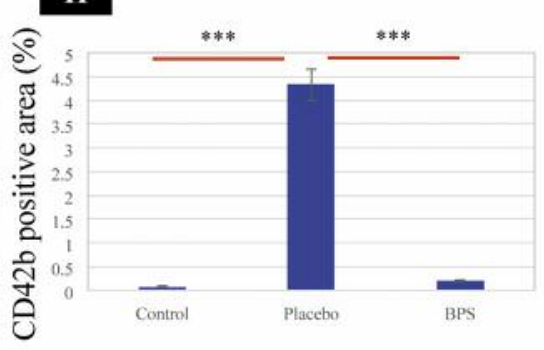

ஓं
I

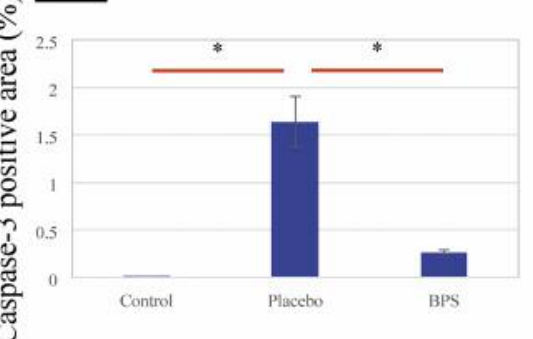

Figure 1. Evaluation of sinusoidal obstruction syndrome and sinusoidal endothelial cells. A, B: Visual examination: Female Crl:CD1(ICR) mice were divided into the following three groups: (i) control group; (ii) placebo group (administered $270 \mathrm{mg} / \mathrm{kg}$ MCT); and (iii) BPS group (administered $270 \mathrm{mg} / \mathrm{kg} \mathrm{MCT}+200 \mu \mathrm{g} / \mathrm{kg}$ BPS). The BPS was administered intraperitoneally $1 \mathrm{~h}$ before, and then both 3 and $9 \mathrm{~h}$ after MCT. The mice were killed 48 h later. Massive bloody ascites and dark red coloration of the liver were found in the placebo group. No ascites was found in the BPS group, and the coloration was also similar to that of the control group. These changes became more marked after perfusion from the portal vein. $C: H \& E$ staining: In the placebo group, sinusoidal dilation and blood congestion were found centered in zone 3. However, these signs were suppressed in the BPS group and were approximately the same as the control group. D, E, F: Immunostaining: SE-1: Staining of the sinusoids in zone 3 was reduced in the placebo group, whereas in the BPS group, there was clear staining along the sinusoids while the LSECs were protected. $C D 42 b$ : In the placebo group, staining was found centered around the space of Disse in zone 3, suggesting EPA; expression was reduced in the BPS group. Caspase-3: In the placebo group, caspase-3 expression was found centered in zone 3, suggesting apoptosis; expression was reduced in the BPS group. G, H, I: Evaluation of immunostaining using image J: Areas of SE-1, CD42b and Caspase-3 staining were evaluated using the average of five randomly selected images on image J. Significant differences were considered at $* p<0.05$, and $* * p<0.01, * * * p<0.001$.

induction of vasodilation; and protective effects of the LSECs.

SOS is a complication that may occur after hematopoietic stem cell transplantation, liver transplantation, or hepatectomy after L-OHP-based chemotherapy and has a very poor prognosis $(4,7,8)$. Although various causes have been suggested for SOS, including LSEC glutathione exhaustion, nitric oxide exhaustion, matrix metalloproteinase increase, and vascular endothelial growth factor (VEGF) increase, its etiology has still not been elucidated (22). Various pharmacological agents are currently under investigation as potential treatments for SOS/VOD. Defibrotide has been reported to be effective and is approved for the treatment of SOS in both Europe and the USA (9), however, its use in Japan is still not permitted. Additionally, it has been suggested that antithrombin III, prostaglandin E1, low-molecular-weight heparin, and ursodeoxycholic acid are effective, although this has not been verified (10-13). Furthermore, there have been 

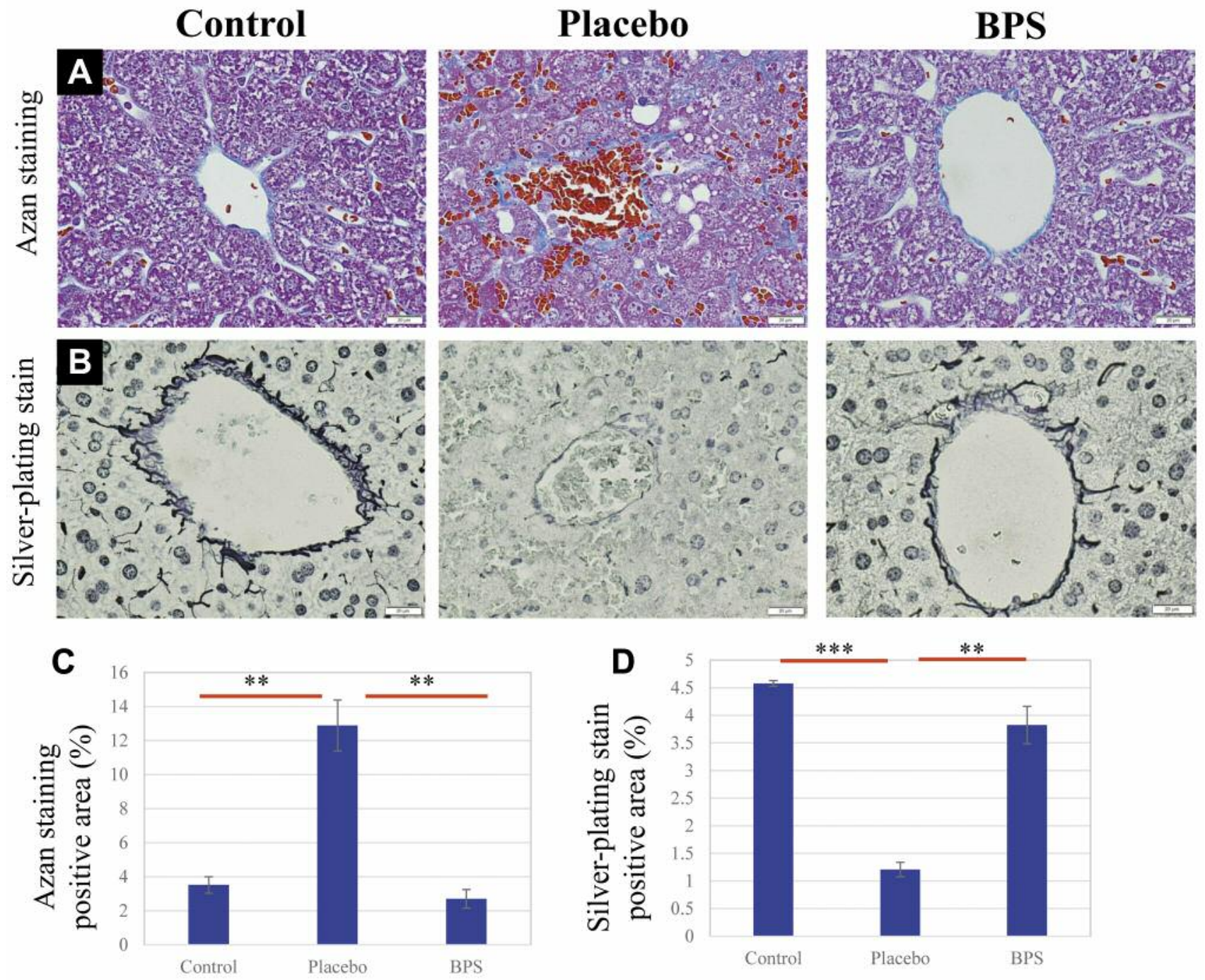

Figure 2. Evaluation of connective tissue fibers around zone 3. A, B: Azan staining and silver-plating stain: Azan staining gave blue-purple staining centered around zone 3 in the placebo group. In the BPS group, black staining centered in zone 3 was observed after silver-plating stain, similarly to the control group. These findings suggest that in the placebo group, reticular fibers (Type III collagen) decreased while collagen fibers (Type I collagen) increased; in the BPS group, however, these changes were alleviated and EPA was inhibited. C, D: Evaluation of Azan staining and silverplating stain using image J: Areas subjected to Azan and silver-plating stains were evaluated using the average of five randomly selected images on image J. Significant differences were considered at $* p<0.05$, and $* * p<0.01$, ***p $<0.001$.

reports indicating that glutathione, matrix-metalloproteinaseinhibitors, phosphodiesterase III-inhibitors, and sorafenib can inhibit SOS in a rat model; however, use of these have not yet reached the clinical application stage (23-25).

The pathology of SOS has not been fully elucidated; in terms of pathological findings, the initial stage of SOS is characterized by sinusoidal dilation in zone 3 surrounding the central vein, hepatocyte necrosis, blood congestion, and intimal edema. Later stages of SOS involve marked peripheral venous fibrosis and collagen deposition $(5,26)$. Onset of this condition may therefore be due to loss of the
LSECs. The authors have previously reported that the principal etiology of SOS is via damage to LSECs; this results in their detachment and destruction, eventually causing organ dysfunction via sepsis $(27,28)$. This may lead to extravascular leakage of platelets, and thus, EPA in the space of Disse and various other pathologies related to platelet-derived factors $(14,16,17)$. Platelets contain $\alpha$ granules and dense granules; upon activation, strong proinflammatory chemokines included in these granules are released (29). These substances are proliferative factors and include TXA2, VEGF-A, TGF- $\beta$, and PAI-1. TXA2 has 


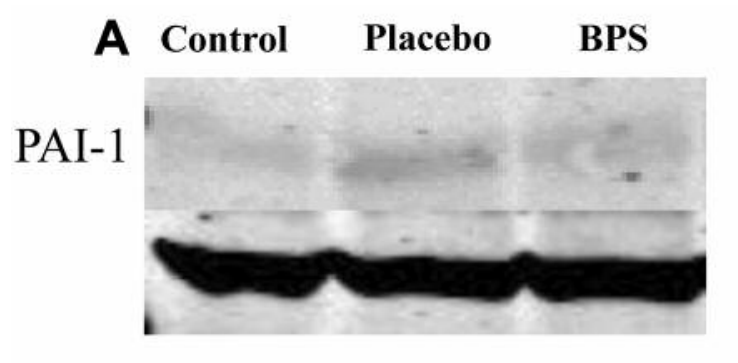

C PAI-1

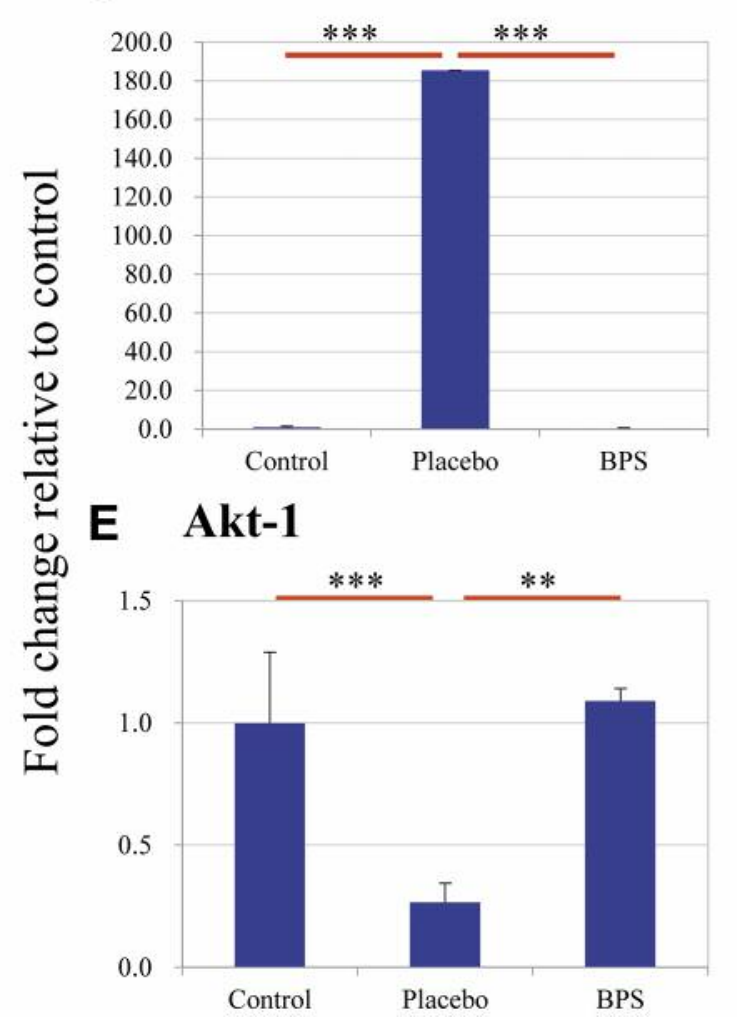

B Control Placebo Placebo BPS BPS

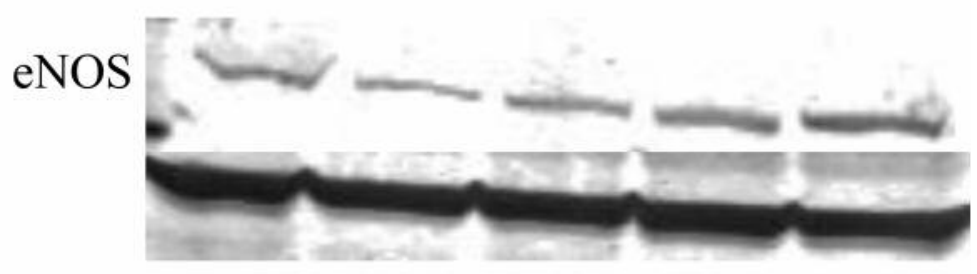

D eNOS
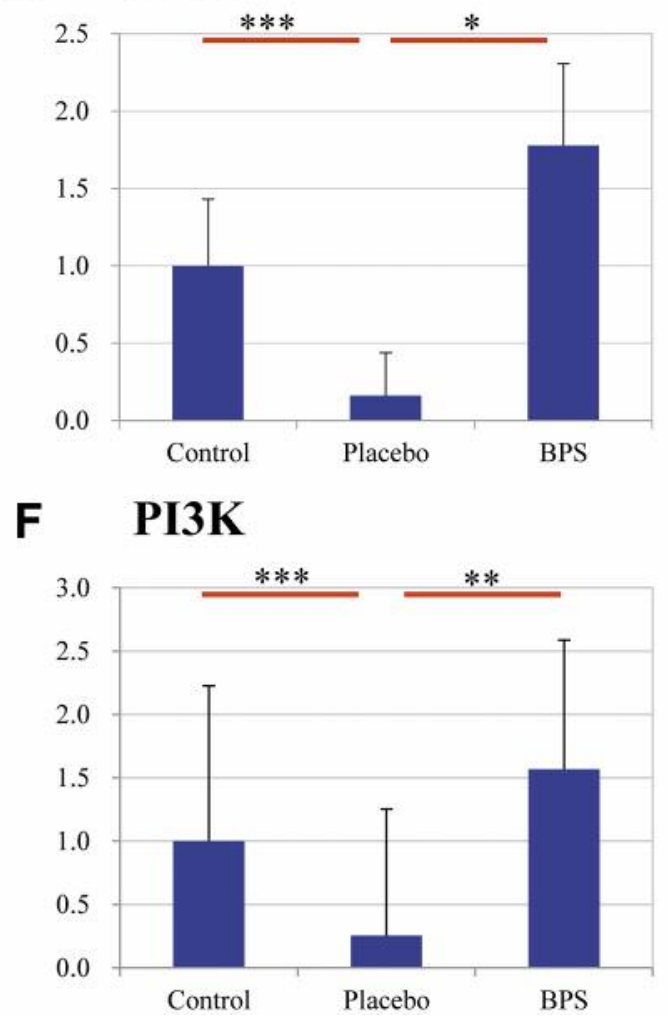

Figure 3. Evaluation of vascular endothelial cell damage. A, B: Western blotting: PAI-1 expression was higher in the placebo group than in the BPS and control groups, while eNOS expression was higher in the BPS group than in the placebo and control groups. C, D, E, F: Real-time RT-PCR: PAI-1 was expressed more markedly in the placebo group than in the BPS and control groups, whereas eNOS, Akt-1 and PI3K were expressed at higher levels in the BPS group than in the placebo and control groups. Significant differences were considered at $* p<0.05$, and $* * p<0.01, * * * p<0.001$.

vasoconstrictive as well as platelet-aggregatory effects and induces portal hypertension by increasing portal resistance (30). VEGF-A is generally known for its vasodilatory effects, but is also used as an agent with opposite effects; vasoconstriction has been reported under conditions of incomplete vascular endothelium (31). It has therefore been reported that administration of bevacizumab, an anti-VEGFA antibody, results in alleviation of SOS induced by oxaliplatin in terms of both incidence and severity (32). TGF- $\beta$ is a strong, fibrosis-promoting cytokine that stimulates collagen synthesis mediated by hepatic stellate cells, as well as inhibits hepatocyte proliferation $(33,34)$. PAI-1 is a marker of vascular endothelial damage, but also inhibits the fibrinolysis reaction by deactivating both tissuetype plasminogen activator (t-PA) and urokinase-type plasminogen activator (u-PA). The hepatocyte proliferation factor is activated by u-PA, while PAI- 1 inhibits u-PA, thus creating a hepatocyte-proliferation-inhibiting factor $(35,36)$. On the basis of the above findings, the authors consider that SOS itself may be caused by extravasated-platelet-derived factors, and that prophylactic treatment by LSEC protection is an important measure for responding to SOS. 
This study was an investigation as to whether BPS, a PGI2 derivative, has therapeutic or prophylactic effects in SOS. PGI2 is produced via prostaglandin endoperoxide by enzymes such as phospholipase A2, cyclooxygenase, and PGI2 synthase (37). According to previous reports, in addition to anti-platelet effects, vasodilatory effects and anti-inflammatory effects (38), PGI2 has vascular endothelial protective, as well as antiapoptotic effects (39). BPS is a synthetic PGI2 analogue that has higher chemical and metabolic stability than PGI2. In a study using a rat model with pulmonary emphysema induced by cigarette smoke extract, Chen et al. demonstrated that administration of BPS reduced caspase- 3 expression, and had an anti-apoptotic effect (40). In addition, BPS binds the IP receptor, and the IP receptor activates Gs, which activates adenylyl cyclase via Gs. Activation of the IP receptor increases the level of cyclic adenosine monophosphate (cAMP), thus inducing vasodilation, and inhibiting platelet function (41). BPS also activates cAMP-dependent protein kinase $\mathrm{A}$, thus inducing nitric oxide production and vasodilation via eNOS phosphorylation (42).

Togawa et al. have reported that BPS, a synthetic PGI2 analogue, induces expression of Akt (i.e. protein kinase B) (43). PGI2 acts as a ligand with a G-protein-coupled receptor (GPCR), thus activating PI3K as well as eNOS via phosphorylation by Akt; the kinase is positioned downstream, resulting in promotion of nitric oxide production $(44,45)$. Regarding insulin acting similarly to a GPCR ligand, Kubota et al. have demonstrated that in obese patients with type-2 diabetes, blockage of insulin signal transmission by endothelial cells reduces insulin-receptor substrate-2 expression, as well as insulin-induced eNOS phosphorylation; this induces the attenuation of insulin-induced capillary recruitment and insulin delivery, reducing glucose uptake via the skeletal muscles (19). Conversely, Kubota et al. have also reported, using a murine model, that recovery of eNOS phosphorylation by endothelial cells has the opposite effect, reducing attenuation of insulin-induced capillary recruitment and insulin delivery and leading to recovery of endothelial cell function and glucose uptake (36). In connection with the PI3K-Akt pathway being linked to fundamental cell processes, including protein synthesis, cell proliferation, and cell survival, activation of this pathway results in eNOS increasing the release of phosphates and nitric oxide (43-45). In addition, activation of the PI3K-Akt-eNOS pathway has been reported to inhibit apoptosis of vascular endothelial cells $(39,46)$.

\section{Conclusion}

BPS administration has been shown to activate the PI3KAkt-eNOS pathway, and to have anti-apoptotic effects on LSECs. It is suggested that LSEC functional enhancement due to BPS administration may inhibit EPA, thus preventing progression to SOS.

\section{Conflicts of Interest}

The Authors report no proprietary or commercial interest in any product mentioned or concept discussed in this article.

\section{Authors' Contributions}

Makoto Nakura, Tomoharu Miyashita and Yasuhiko Yamamoto made substantial contributions to the design and coordination of the study. Makoto Nakura, Satoshi Takada and Shunsuke Kanou carried out the immunohistochemistry. Makoto Nakura, Hidehiro Tajima and Hiroyuki Takamura collected the samples and data. Makoto Nakura and Tomoharu Miyashita analyzed the data. Tetsuo Ohta approved the final version of the manuscript. All Authors read and approved the final manuscript.

\section{References}

1 DeLeve LD, Shulman HM and McDonald GB: Toxic injury to hepatic sinusoids: Sinusoidal obstruction syndrome (venoocclusive disease). Semin Liver Dis 22(1): 27-42, 2002. PMID: 11928077. DOI: $10.1055 / \mathrm{s}-2002-23204$

2 Campos-Varela I, Castells L, Dopazo C, Pérez-Lafuente M, Allende $\mathrm{H}$, Len O, Llopart L, Vargas V and Charco R: Transjugular intrahepatic portosystemic shunt for the treatment of sinusoidal obstruction syndrome in a liver transplant recipient and review of the literature. Liver Transpl 18(2): 201-205, 2012. PMID: 21656652. DOI: 10.1002/lt.22351

3 McDonald GB, Hinds MS, Fisher LD, Schoch HG, Wolford JL, Banaji M, Hardin BJ, Shulman HM and Clift RA: Venoocclusive disease of the liver and multiorgan failure after bone marrow transplantation: A cohort study of 355 patients. Ann Intern Med 118(4): 255-267, 1993. PMID: 8355040. DOI: 10.1200/JCO.1993.11.9.1729

4 Coppell JA, Richardson PG, Soiffer R, Martin PL, Kernan NA, Chen A, Guinan E, Vogelsang G, Krishnan A, Giralt S, Revta C, Carreau NA, Iacobelli M, Carreras E, Ruutu T, Barbui T, Antin $\mathrm{JH}$ and Niederwieser D: Hepatic veno-occlusive disease following stem cell transplantation: Incidence, clinical course, and outcome. Biol Blood Marrow Transplant 16(2): 157-168, 2010. PMID: 19766729. DOI: 10.1016/j.bbmt.2009.08.02

5 Fan CQ and Crawford JM: Sinusoidal obstruction syndrome (hepatic veno-occlusive disease). J Clin Exp Hepatol 4(4): 332346, 2014. PMID: 25755580. DOI: 10.1016/j.jceh.2014.10.002

6 Tajima H, Ohta T, Miyashita T, Nakanuma S, Matoba M, Miyata T, Sakai S, Okamoto K, Makino I, Kinoshita J, Hayashi H, Nakamura K, Oyama K, Inokuchi M, Nakagawara H, Takamura H, Kitagawa $\mathrm{H}$, Fushida S and Ikeda H: Oxaliplatin-based chemotherapy induces extravasated platelet aggregation in the liver. Mol Clin Oncol 3(3): 555-558, 2015. PMID: 26137266. DOI: 10.3892/mco.2015.512

7 Soubrane O, Brouquet A, Zalinski S, Terris B, Brézault C, Mallet V, Goldwasser F and Scatton O: Predicting high grade lesions of sinusoidal obstruction syndrome related to oxaliplatinbased chemotherapy for colorectal liver metastases: Correlation with post-hepatectomy outcome. Ann Surg 251(3): 454-460, 2010. PMID: 20160638. DOI: 10.1097/SLA.0b013e3181c79403

8 Takamura H, Nakanuma S, Hayashi H, Tajima H, Kakinoki K, Kitahara M, Sakai S, Makino I, Nakagawara H, Miyashita T, Okamoto K, Nakamura K, Oyama K, Inokuchi M, Ninomiya I, 
Kitagawa H, Fushida S, Fujimura T, Onishi I, Kayahara M, Tani $\mathrm{T}$, Arai K, Yamashita T, Kitamura H, Ikeda H, Kaneko S, Nakanuma Y, Matsui $\mathrm{O}$ and Ohta T: Severe veno-occlusive disease/sinusoidal obstruction syndrome after deceased-donor and living-donor liver transplantation. Transplant Proc 46(10): 35233535, 2014. PMID: 25498084. DOI: 10.1016/j.transproceed. 2014.09.110

9 Baker DE and Demaris K: Defibrotide. Hosp Pharm 51(10): 847-854, 2016. PMID: 27928191. DOI: $10.1310 / \mathrm{hpj5110-847}$

10 Peres E, Kintzel P, Dansey R, Baynes R, Abidi M, Klein J, Ibrahim RB and Abella E: Early intervention with antithrombin III therapy to prevent progression of hepatic venoocclusive disease. Blood Coagul Fibrinolysis 19(3): 203-207, 2008. PMID: 18388499. DOI: 10.1097/MBC.0b013e3282f2b5d9

11 Inukai T, Sugita K, Goi K, Akahane K, Hirose K, Nemoto A, Takahashi K, Sato H, Uno K, Furuichi Y, Nakamura M, Miyamoto N, Yamakawa N, Shiraishi K, Kojika S, Tezuka T, Iijima $\mathrm{K}$ and Nakazawa S: [Prevention of hepatic veno-occlusive disease by a combination of heparin and prostaglandin e 1 in children undergoing hematopoietic stem cell transplantation]. Rinsho Ketsueki 45(4): 297-303, 2004. PMID: 15168445.

12 Attal M, Huguet F, Rubie H, Huynh A, Charlet JP, Payen JL, Voigt JJ, Brousset P, Selves J and Muller C: Prevention of hepatic veno-occlusive disease after bone marrow transplantation by continuous infusion of low-dose heparin: A prospective, randomized trial. Blood 79(11): 2834-2840, 1992. PMID: 1586733.

13 Ohashi K, Tanabe J, Watanabe R, Tanaka T, Sakamaki H, Maruta A, Okamoto S, Aotsuka N, Saito K, Nishimura M, Oh H, Matsuzaki M, Takahashi S and Yonekura S: The japanese multicenter open randomized trial of ursodeoxycholic acid prophylaxis for hepatic veno-occlusive disease after stem cell transplantation. Am J Hematol 64(1): 32-38, 2000. PMID: 10815785. DOI: $10.1002 /$ (sici)1096-8652(200005)64:1<32::aidajh6>3.0.co;2-n

14 Miyashita T, Nakanuma S, Ahmed AK, Makino I, Hayashi H, Oyama K, Nakagawara H, Tajima H, Takamura H, Ninomiya I, Fushida S, Harmon JW and Ohta T: Ischemia reperfusionfacilitated sinusoidal endothelial cell injury in liver transplantation and the resulting impact of extravasated platelet aggregation. European Surgery-Acta Chirurgica Austriaca 48(2): 92-98, 2016. PMID: 27110233. DOI: 10.1007/s10353-015-0363-3

15 Nakanuma S, Miyashita T, Hayashi H, Tajima H, Takamura H, Tsukada T, Okamoto K, Sakai S, Makino I, Kinoshita J, Nakamura K, Oyama K, Inokuchi M, Nakagawara H, Ninomiya I, Kitagawa H, Fushida S, Fujimura T and Ohta T: Extravasated platelet aggregation in liver zone 3 may correlate with the progression of sinusoidal obstruction syndrome following living donor liver transplantation: A case report. Exp Ther Med 9(4): 1119-1124, 2015. PMID: 25780397. DOI: 10.3892/etm.2015.2245

16 Hirata M, Tajima H, Miyashita T, Miyata T, Nakanuma S, Makino I, Hayashi H, Oyama K, Takamura H, Ninomiya I, Fushida S, Nakata H, Iseki S, Harada S, Wakayama T and Ohta $\mathrm{T}$ : Extravasated platelet aggregation in the livers of rats with drug-induced hepatic sinusoidal obstruction syndrome. Mol Med Rep 15(5): 3147-3152, 2017. PMID: 28358421. DOI: 10.3892/mmr.2017.6407

17 Miyata T, Tajima H, Hirata M, Nakanuma SI, Makino I, Hayashi H, Oyama K, Miyashita T, Takamura H, Ninomiya I, Fushida S, Iseki S, Harada SI, Wakayama T and Ohta T: Phosphodiesterase
III inhibitor attenuates rat sinusoidal obstruction syndrome through inhibition of platelet aggregation in disse's space. J Gastroenterol Hepatol 33(4): 950-957, 2018. PMID: 28960464. DOI: $10.1111 /$ jgh.14004

18 Takada S, Miyashita T, Yamamoto Y, Kanou S, Munesue S, Ohbatake Y, Nakanuma S, Okamoto K, Sakai S, Kinoshita J, Makino I, Nakamura K, Tajima H, Takamura H, Ninomiya I, Fushida $\mathrm{S}$ and Ohta T: Soluble thrombomodulin attenuates endothelial cell damage in hepatic sinusoidal obstruction syndrome. In Vivo 32(6): 1409-1417, 2018. PMID: 30348695. DOI: 10.21873 /invivo.11393

19 Kubota T, Kubota N, Kumagai H, Yamaguchi S, Kozono H, Takahashi T, Inoue M, Itoh S, Takamoto I, Sasako T, Kumagai K, Kawai T, Hashimoto S, Kobayashi T, Sato M, Tokuyama K, Nishimura S, Tsunoda M, Ide T, Murakami K, Yamazaki T, Ezaki O, Kawamura K, Masuda H, Moroi M, Sugi K, Oike Y, Shimokawa H, Yanagihara N, Tsutsui M, Terauchi Y, Tobe K, Nagai R, Kamata K, Inoue K, Kodama T, Ueki K and Kadowaki T: Impaired insulin signaling in endothelial cells reduces insulininduced glucose uptake by skeletal muscle. Cell Metab 13(3): 294307, 2011. PMID: 21356519. DOI: 10.1016/j.cmet.2011.01.018

20 Sakai A, Yajima M and Nishio S: Cytoprotective effect of trk100 , a prostacyclin analogue, against chemical injuries in cultured human vascular endothelial cells. Life Sci 47(8): 711-719, 1990. PMID: 2119471. DOI: 10.1016/0024-3205(90)90626-3

21 Narita M, Hatano E, Ikai I, Miyagawa-Hayashino A, Yanagida A, Nagata $H$, Asechi $H$, Taura $K$ and Uemoto S: A phosphodiesterase III inhibitor protects rat liver from sinusoidal obstruction syndrome through heme oxygenase-1 induction. Ann Surg 249(5): 806-813, 2009. PMID: 19387321. DOI: 10.1097/SLA.0b013e3181a38ed5

22 Helmy A: Review article: Updates in the pathogenesis and therapy of hepatic sinusoidal obstruction syndrome. Aliment Pharmacol Ther 23(1): 11-25, 2006. PMID: 16393276. DOI: 10.1111/j.1365-2036.2006.02742.x

23 DeLeve LD, Ito Y, Bethea NW, McCuskey MK, Wang X and McCuskey RS: Embolization by sinusoidal lining cells obstructs the microcirculation in rat sinusoidal obstruction syndrome. Am J Physiol Gastrointest Liver Physiol 284(6): G1045-1052, 2003. PMID: 12584111. DOI: 10.1152/ajpgi.00526.2002

24 Deleve LD, Wang X, Tsai J, Kanel G, Strasberg S and Tokes ZA: Sinusoidal obstruction syndrome (veno-occlusive disease) in the rat is prevented by matrix metalloproteinase inhibition. Gastroenterology 125(3): 882-890, 2003. PMID: 12949732. DOI: 10.1016/s0016-5085(03)01056-4

25 Nakamura K, Hatano E, Narita M, Miyagawa-Hayashino A, Koyama Y, Nagata H, Iwaisako K, Taura K and Uemoto S: Sorafenib attenuates monocrotaline-induced sinusoidal obstruction syndrome in rats through suppression of jnk and mmp-9. J Hepatol 57(5): 1037-1043, 2012. PMID: 22796153. DOI: $10.1016 /$ j.jhep.2012.07.004

26 Livertox: Clinical and research information on drug-induced liver injury. National Institute of Diabetes and Digestive and Kidney Diseases. Bethesda (MD), 2012. PMID: 31643176.

27 Miyashita T, Ahmed AK, Nakanuma S, Okamoto K, Sakai S, Kinoshita J, Makino I, Nakamura K, Hayashi H, Oyama K, Tajima H, Takamura H, Ninomiya I, Fushida S, Harmon JW and Ohta T: A three-phase approach for the early identification of acute lung injury induced by severe sepsis. In Vivo 30(4): 341349, 2016. PMID: 27381595. 
28 Sakurai K, Miyashita T, Okazaki M, Yamaguchi T, Ohbatake Y, Nakanuma S, Okamoto K, Sakai S, Kinoshita J, Makino I, Nakamura K, Hayashi H, Oyama K, Tajima H, Takamura H, Ninomiya I, Fushida S, Harada K, Harmon JW and Ohta T: Role for neutrophil extracellular traps (nets) and platelet aggregation in early sepsis-induced hepatic dysfunction. In Vivo 31(6): 10511058, 2017. PMID: 29102925. DOI: 10.21873/invivo.11169

29 Badimón L, Vilahur G and Padró T: Lipoproteins, platelets and atherothrombosis. Rev Esp Cardiol 62(10): 1161-1178, 2009. PMID: 19793522. DOI: 10.1016/s1885-5857(09)73331-6

30 Ruan Z, Shibamoto T, Shimo T, Koizumi T, Tsuchida H, Kurata Y, Ogura T and Kubo K: Effects of platelet-activating factor and thromboxane a2 on isolated perfused guinea pig liver. Prostaglandins Other Lipid Mediat 73(1-2): 73-85, 2004. PMID: 15165033. DOI: 10.1016/j.prostaglandins.2003.11.002

31 Parenti A, Brogelli L, Filippi S, Donnini S and Ledda F: Effect of hypoxia and endothelial loss on vascular smooth muscle cell responsiveness to vegf-a: Role of flt-1/vegf-receptor- 1 . Cardiovasc Res 55(1): 201-212, 2002. PMID: 12062723. DOI: 10.1016/s0008-6363(02)00326-7

32 Ribero D, Wang H, Donadon M, Zorzi D, Thomas MB, Eng C, Chang DZ, Curley SA, Abdalla EK, Ellis LM and Vauthey JN: Bevacizumab improves pathologic response and protects against hepatic injury in patients treated with oxaliplatin-based chemotherapy for colorectal liver metastases. Cancer 110(12): 2761-2767, 2007. PMID: 17960603. DOI: 10.1002/cncr.23099

33 Ueda S, Yamanoi A, Hishikawa Y, Dhar DK, Tachibana M and Nagasue N: Transforming growth factor-beta1 released from the spleen exerts a growth inhibitory effect on liver regeneration in rats. Lab Invest 83(11): 1595-1603, 2003. PMID: 14615413. DOI: $10.1097 / 01.1 \mathrm{ab} .0000095686 .10639 . \mathrm{c} 8$

34 Russell WE, Coffey RJ, Ouellette AJ and Moses HL: Type beta transforming growth factor reversibly inhibits the early proliferative response to partial hepatectomy in the rat. Proc Natl Acad Sci USA 85(14): 5126-5130, 1988. PMID: 3164865. DOI: 10.1073/pnas.85.14.5126

35 Mars WM, Zarnegar R and Michalopoulos GK: Activation of hepatocyte growth factor by the plasminogen activators upa and tpa. Am J Pathol 143(3): 949-958, 1993. PMID: 8362987.

36 Watanabe K, Togo S, Takahashi T, Matsuyama R, Yamamoto H, Shimizu T, Makino H, Matsuo K, Morioka D, Kubota T, Nagashima Y and Shimada H: Pai-1 plays an important role in liver failure after excessive hepatectomy in the rat. J Surg Res 143(1): 13-19, 2007. PMID: 17655862. DOI: 10.1016/j.jss.2007.04.041

37 Spisni E, Bartolini G, Orlandi M, Belletti B, Santi S and Tomasi V: Prostacyclin (pgi2) synthase is a constitutively expressed enzyme in human endothelial cells. Exp Cell Res 219(2): 507513, 1995. PMID: 7641804. DOI: 10.1006/excr.1995.1259

38 Miyata M, Ueno Y, Sekine H, Ito O, Sakuma F, Koike H, Nishio S, Nishimaki T and Kasukawa R: Protective effect of beraprost sodium, a stable prostacyclin analogue, in development of monocrotaline-induced pulmonary hypertension. J Cardiovasc Pharmacol 27(1): 20-26, 1996. PMID: 8656653. DOI: 10.1097/ 00005344-199601000-00004
39 Chen Y, Luo H, Kang N, Guan C, Long Y, Cao J, Shen Q, Li J, Yang $\mathrm{M}$, Peng $\mathrm{H}$ and Chen $\mathrm{P}$ : Beraprost sodium attenuates cigarette smoke extract-induced apoptosis in vascular endothelial cells. Mol Biol Rep 39(12): 10447-10457, 2012. PMID: 23053949. DOI: 10.1007/s11033-012-1924-1

40 Chen Y, Hanaoka M, Chen P, Droma Y, Voelkel NF and Kubo $\mathrm{K}$ : Protective effect of beraprost sodium, a stable prostacyclin analog, in the development of cigarette smoke extract-induced emphysema. Am J Physiol Lung Cell Mol Physiol 296(4): L648656, 2009. PMID: 19201816. DOI: 10.1152/ajplung.90270.2008

41 Tanaka Y, Yamaki F, Koike K and Toro L: New insights into the intracellular mechanisms by which pgi2 analogues elicit vascular relaxation: Cyclic amp-independent, gs-protein mediatedactivation of maxik channel. Curr Med Chem Cardiovasc Hematol Agents 2(3): 257-265, 2004. PMID: 15320791. DOI: $10.2174 / 1568016043356273$

42 Ono S, Nagaoka T, Omae T, Tanano I, Kamiya T, Otani S, Ishibazawa A and Yoshida A: Beraprost sodium, a stable prostacyclin analogue, elicits dilation of isolated porcine retinal arterioles: Roles of enos and potassium channels. Invest Ophthalmol Vis Sci 55(9): 5752-5759, 2014. PMID: 25082887. DOI: $10.1167 /$ iovs.14-14902

43 Togawa M, Haneda M, Araki S, Sugimoto T, Isono M, Hidaka $\mathrm{H}$, Yasuda H, Kashiwagi A and Kikkawa R: Beraprost sodium, an analogue of prostacyclin, induces the expression of mitogenactivated protein kinase phosphatase and inhibits the proliferation of cultured mesangial cells. Eur J Pharmacol 336(23): 291-294, 1997. PMID: 9384245. DOI: 10.1016/s00142999(97)01208-9

44 Zeng G, Nystrom FH, Ravichandran LV, Cong LN, Kirby M, Mostowski H and Quon MJ: Roles for insulin receptor, pi3kinase, and akt in insulin-signaling pathways related to production of nitric oxide in human vascular endothelial cells. Circulation 101(13): 1539-1545, 2000. PMID: 10747347. DOI: 10.1161/01.cir.101.13.1539

45 Eyster KM: The membrane and lipids as integral participants in signal transduction: Lipid signal transduction for the non-lipid biochemist. Adv Physiol Educ 31(1): 5-16, 2007. PMID: 17327576. DOI: 10.1152/advan.00088.2006

46 Cutler NS, Graves-Deal R, LaFleur BJ, Gao Z, Boman BM, Whitehead RH, Terry E, Morrow JD and Coffey RJ: Stromal production of prostacyclin confers an antiapoptotic effect to colonic epithelial cells. Cancer Res 63(8): 1748-1751, 2003. PMID: 12702555.
Received July 13, 2020

Revised August 1, 2020 Accepted August 3, 2020 